\title{
Selective dip-coating of chemically micropatterned surfaces
}

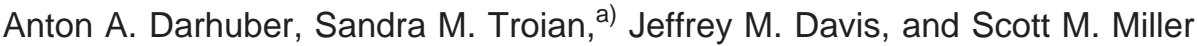 \\ Interfacial Science Laboratory, Dept. of Chemical Engineering, Princeton University, Princeton, \\ New Jersey 08544 \\ Sigurd Wagner \\ Department of Electrical Engineering, Princeton University, Princeton, New Jersey 08544
}

(Received 11 May 2000; accepted for publication 17 August 2000)

\begin{abstract}
We characterize the selective deposition of liquid microstructures on chemically heterogeneous surfaces by means of dip coating processes. The maximum deposited film thickness depends critically on the speed of withdrawal as well as the pattern size, geometry, and angular orientation. For vertically oriented hydrophilic strips, we derive a hydrodynamic scaling relation for the deposited film thickness which agrees very well with interferometric measurements of dip-coated liquid lines. Due to the lateral confinement of the liquid, our scaling relation differs considerably from the classic Landau-Levich formula for chemically homogeneous surfaces. Dip coating is a simple method for creating large area arrays of liquid microstructures for applications involving chemical analysis and synthesis, biochemical assays, or wet printing of liquid polymer or ink patterns. (C) 2000 American Institute of Physics. [S0021-8979(00)07122-X]
\end{abstract}

\section{INTRODUCTION}

Thin liquid films are commonly deposited on planar or cylindrical surfaces by a dip-coating process. This technique is widely used in industrial applications because of its simplicity and high throughput. A flat or curved substrate, which is normally smooth and wettable by the liquid coating, is withdrawn at constant velocity from a liquid reservoir. Depending on liquid composition, layered coatings can be deposited in this way in a controlled manner. The challenge to this technique, especially at higher withdrawal speed, is to form a smooth coating without defects like blisters, holes, cracks, or local material accumulation. ${ }^{1-3}$

With the aid of photolithography or microcontact printing, ${ }^{4,5}$ the wetting properties of surfaces can be tailored with submicron resolution. Molecules like alkylsilanes or alkylthiols form so-called self-assembled monolayers which render a hydrophilic surface like silicon dioxide or gold hydrophobic. This is reflected, for example, in the large increase of the contact angle of water from $0^{\circ}$ to about $110^{\circ}$. By patterning such a hydrophobic monolayer, ultrasmall volumes of liquid in the nanoliter to femtoliter range can be precisely distributed at desired locations by dip coating the substrate into a liquid bath or slot. Such small volume distributions are difficult or even impossible to attain with conventional dispensing techniques like ink jetting or microsyringe delivery.

The dip coating of plates, cylinders, and fibers has been investigated for over six decades. The majority of experimental and theoretical studies ${ }^{6-18}$ have focused exclusively on chemically homogeneous surfaces. As the number of techniques available for microfabrication and micropatterning grows, there is interest in selectively coating or "inking"

\footnotetext{
a) Author to whom correspondence should be addressed; electronic mail:
} stroian@princeton.edu chemically patterned surfaces for use as chemical microreactors. Biebuyck and Whitesides have used an immersion coating technique to fabricate microlens arrays on a chemically patterned surface. ${ }^{19}$ Qin et al. ${ }^{20}$ applied a similar technique to fabricate $\mathrm{CuSO}_{4}$ and $\mathrm{KNO}_{3}$ microcrystals from aqueous solutions. Braun and Meyer ${ }^{21}$ have produced a structured thin polymer film by dip coating an array of water droplets on a hydrophilized gold surface. These approaches provide a simple yet elegant method for selective material deposition.

In this article, we investigate the dip coating of chemically micropatterned surfaces. Besides the liquid material properties like the viscosity, surface tension, and density, the thickness of the liquid coating which adheres to the hydrophilic portions depends critically on the pattern size, geometry and orientation. Since our application of interest is the "printing" of liquid micropatterns onto a secondary target surface, we require pattern fidelity between the designed chemical pattern and the liquid microstructures formed, as well as a uniform coating thickness across structures of varying size or shapes. In addition to these experimental investigations, we derive a hydrodynamic model for the maximum film height deposited on vertically oriented hydrophilic strips on a hydrophobic plane. The predictions of this model, which differs in two key ways from the traditional dipcoating analysis on homogeneous surfaces, agree remarkably well with experimental results.

\section{EXPERIMENTAL SETUP}

The samples were prepared from [001]-oriented $p$-type doped silicon wafers using optical lithography. The wafers were first cleaned by immersion in a solution of concentrated sulfuric acid and hydrogen peroxide (volume ratio of 7-3) at $90^{\circ} \mathrm{C}$ for $15 \mathrm{~min}$, then thoroughly rinsed in ultrapure, deionized water $(18 \mathrm{M} \Omega$ ). Subsequently, thin layers of $\mathrm{Cr}$ (5 $\mathrm{nm})$ and $\mathrm{Au}(40 \mathrm{~nm})$ were deposited with an electron beam 
evaporator. A layer of photoresist was spin coated onto the metal layers, which were then patterned by optical lithography and wet chemical etching using TFA (Transene Comp., Inc.) and CR-7 (Cyantek Corp.). After stripping off the photoresist, the samples were immersed in a $1 \mathrm{mM}$ solution of hexadecanethiol (HDT) in pure ethanol at a temperature of $30^{\circ} \mathrm{C}$ for $45 \mathrm{~min}$. The thiol end groups of the HDT molecules bond to the gold forming a hydrophobic self-assembled monolayer since the alkane chains orient away from the gold surface. The contact angle of water on HDT was measured to be $108^{\circ} \pm 3^{\circ}$.

Regions where the gold and chromium layers are removed and the silicon-dioxide exposed are hydrophilic with a contact angle below $5^{\circ}$. If the samples are exposed to ambient air, the contact angle on the hydrophilic parts increases over several days. This is likely due to organic contamination of silicon-dioxide, which is known to have a high surface energy.

The masks used for the optical lithography of the samples were printed on a transparent polymer foil by a high-resolution image setter. The minimum feature size was approximately $25 \mu \mathrm{m}$. Smaller spacings between individual elements could not be resolved by the image setter.

The liquid used in this study was glycerol (1,2,3 trihydroxy propane, $\mathrm{C}_{3} \mathrm{H}_{8} \mathrm{O}_{3}$ ). It has a very low vapor pressure such that evaporation could be held to a minimum, an important consideration given the small scale structures formed. Glycerol is hygroscopic, however, and the absorption of water reduces the viscosity significantly. The viscosity was measured with a capillary viscometer as 0.975 $\pm 0.060 \mathrm{Pas}$ at $22^{\circ} \mathrm{C}$, the surface tension of glycerol is $0.0634 \mathrm{~N} / \mathrm{m}$ at $20^{\circ} \mathrm{C}^{22}$

The apparatus used for dip coating the patterned silicon wafers consisted of a computer controlled sample stage, which allows for precise control of the speed of withdrawal. The direction of withdrawal with respect to the liquid reservoir and the in-plane orientation of the sample were controlled with a precision of approximately $\pm 2^{\circ}$. Film thickness profiles of the deposited liquid microstructures were measured by optical microscopy (Olympus BX60) using a green bandpass filter whose transmission band was centered about $550 \mathrm{~nm}$.

\section{THEORETICAL DESCRIPTION}

\section{A. Dip-coating of homogeneous surfaces}

Landau and Levich ${ }^{6,7}$ and Deryagin ${ }^{8}$ were the first to calculate the maximum thickness of a film, $h_{\infty}$, entrained on an infinite flat plate withdrawn vertically from a reservoir of a Newtonian liquid. In their derivation, they assumed that gravitational drainage was negligible and that the coating thickness was established by a balance between viscous and capillary forces. Their expression, obtained in the lubrication approximation, which essentially requires laminar and small aspect ratio flow, ${ }^{23}$ is given by

$$
h_{\infty}=0.946 \sqrt{\frac{\sigma}{\rho g}} \mathrm{Ca}^{2 / 3} .
$$

As discussed by Wilson, ${ }^{14}$ this result is only valid for low capillary numbers, $\mathrm{Ca}=\mu U / \sigma \ll 1$, since the viscous contribution to the normal pressure is neglected in the derivation. Here, $U$ is the plate withdrawal speed from liquid reservoir and $\mu, \sigma$, and $\rho$ denote the liquid viscosity, surface tension, and density, respectively. The key point in obtaining this expression relies on matching the flat film profile above the reservoir to the profile of a static meniscus. ${ }^{7}$ The characteristic length, $l_{c}=\sqrt{\sigma / 2 \rho g}$, which determines the thickness of the entrained film, represents the radius of curvature of a static capillary meniscus on a completely wetting wall or likewise the height of capillary rise. For different geometries like the dip coating of a very fine fiber, for which the meniscus radius of curvature is much larger than the fiber radius, the relevant length scale becomes the fiber radius since its associated capillary pressure dominates the flow. ${ }^{18}$ The corresponding prefactor in Eq. (1) also changes slightly but remains of order one.

Modifications by White and Tallmadge ${ }^{10}$ and Spiers et al. ${ }^{13}$ extended the applicability of this model toward larger values of $\mathrm{Ca}$. Wilson ${ }^{14}$ later corrected these two analyses and derived an expression valid to second order in $\mathrm{Ca}$ by using the method of matched expansions

$$
\begin{aligned}
h_{\infty}= & \sqrt{\frac{\sigma}{\rho g}} \frac{1}{\sqrt{1-\sin \alpha}} \\
& \times\left[0.94581 \mathrm{Ca}^{2 / 3}-\frac{0.10685 \cos \alpha}{1-\sin \alpha} \mathrm{Ca}\right],
\end{aligned}
$$

where $\alpha$ is the angle of inclination as illustrated in Fig. 1(a). The film thickness predicted by Eq. (2) as a function of the speed of withdrawal $U$ and the angle of inclination $\alpha$ for pure glycerol ( $\mu=1.760 \mathrm{Pas})$ is plotted in Figs. 1(b) and 1(c). More recent numerical studies of the dip-coating process have been presented by Tanguy et al. ${ }^{15}$ and Reglat et al. ${ }^{16}$ Schunk et al. ${ }^{17}$ have extended the model to include mass loss due to solvent evaporation within the framework of a one- and two-phase flow model relevant to sol-gel processing.

\section{B. Capillary rise on a heterogeneous surface}

As outlined in the previous section, the curvature of the (vertical) static meniscus has a decisive influence on the entrained film thickness. When dip-coating patterned surfaces, like vertically oriented hydrophilic strips, however, there is a second curvature in the direction transverse to the liquid ribbon adhering to the hydrophilic strip. As we will show, the transverse curvature depresses the radius of curvature in the vertical direction and thus the entrained film thickness. This allows for the deposition of much thinner liquid films.

We conducted energy minimization calculations ${ }^{24}$ of the shape of the static meniscus on an isolated vertical hydrophilic strip as shown in Fig. 2(a). As the input parameters for the computations, we used the material constants of glycerol and equilibrium contact angles of $5^{\circ}$ and $95^{\circ}$ on the hydrophilic and the hydrophobic regions, respectively. The computational domain had dimensions of $12 \times 12 \mathrm{~mm}^{2}$, which is much larger than the capillary length $\sqrt{\sigma / \rho g}=2.26 \mathrm{~mm}$. 

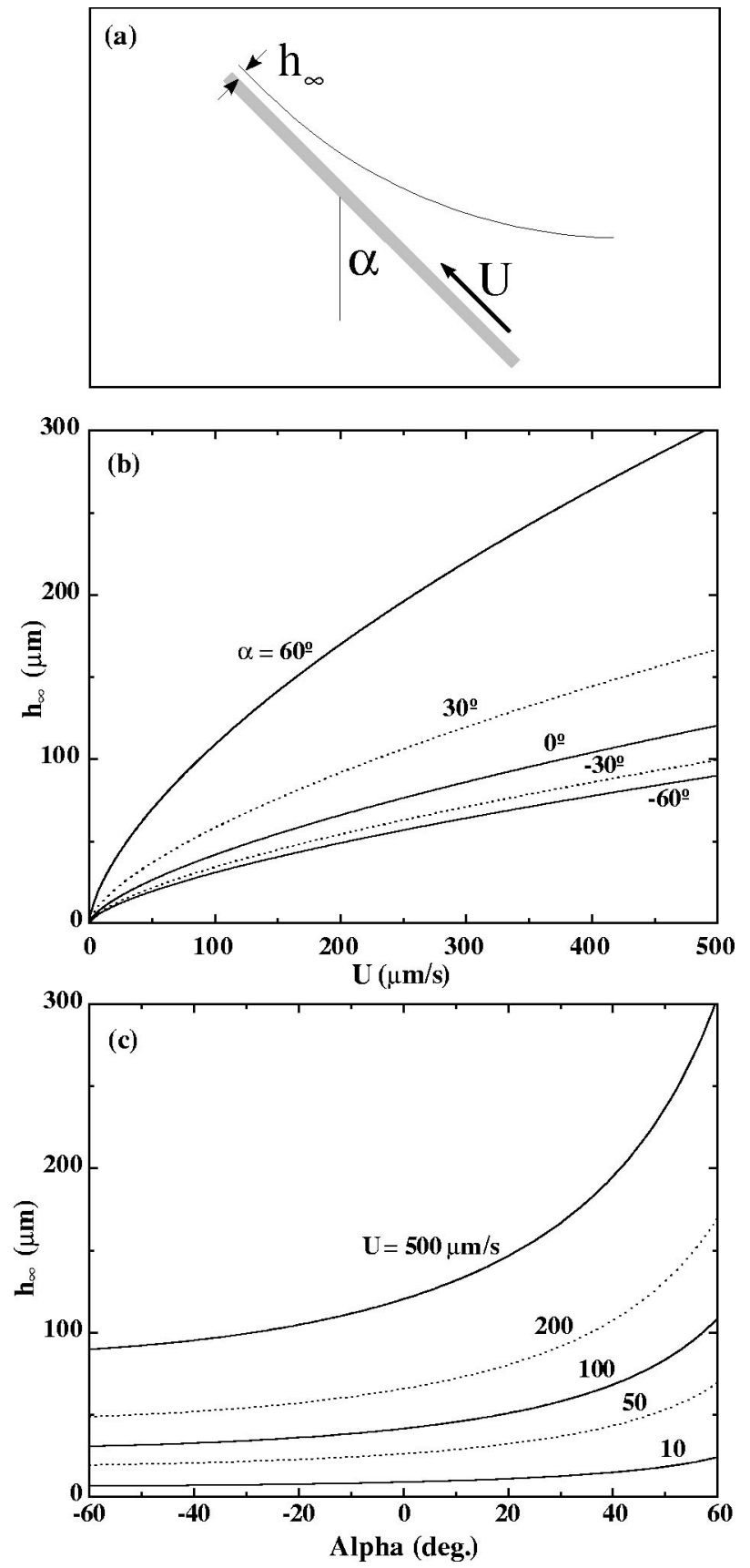

FIG. 1. (a) Sketch of the dip-coating geometry for homogeneous substrates. Entrained film thickness of glycerol as a function of (b) the speed of withdrawal $U$ and (c) the inclination angle $\alpha$ on a homogeneous plate.

Figure 2(a) shows a front view of the static meniscus profile on a $312 \mu \mathrm{m}$ wide isolated hydrophilic line. In Fig. 2(b), we compare various cross sections of the calculated surface profiles through a vertical plane at the center of the hydrophilic line for a series of six linewidths ranging from 78.1 to $2500 \mu \mathrm{m}$. For linewidths large compared to $l_{c}$, the meniscus profile is indistinguishable from that formed on an infinite, uniformly hydrophilic plane surface. For linewidths much smaller than $l_{c}$, the meniscus shape away from the wall is depressed downward, similar to the behavior of a hydrophilic liquid against an infinite, uniformly hydrophobic plane. In the immediate neighborhood of the wall, the liquid senses the chemical heterogeneity and wets the hydrophilic
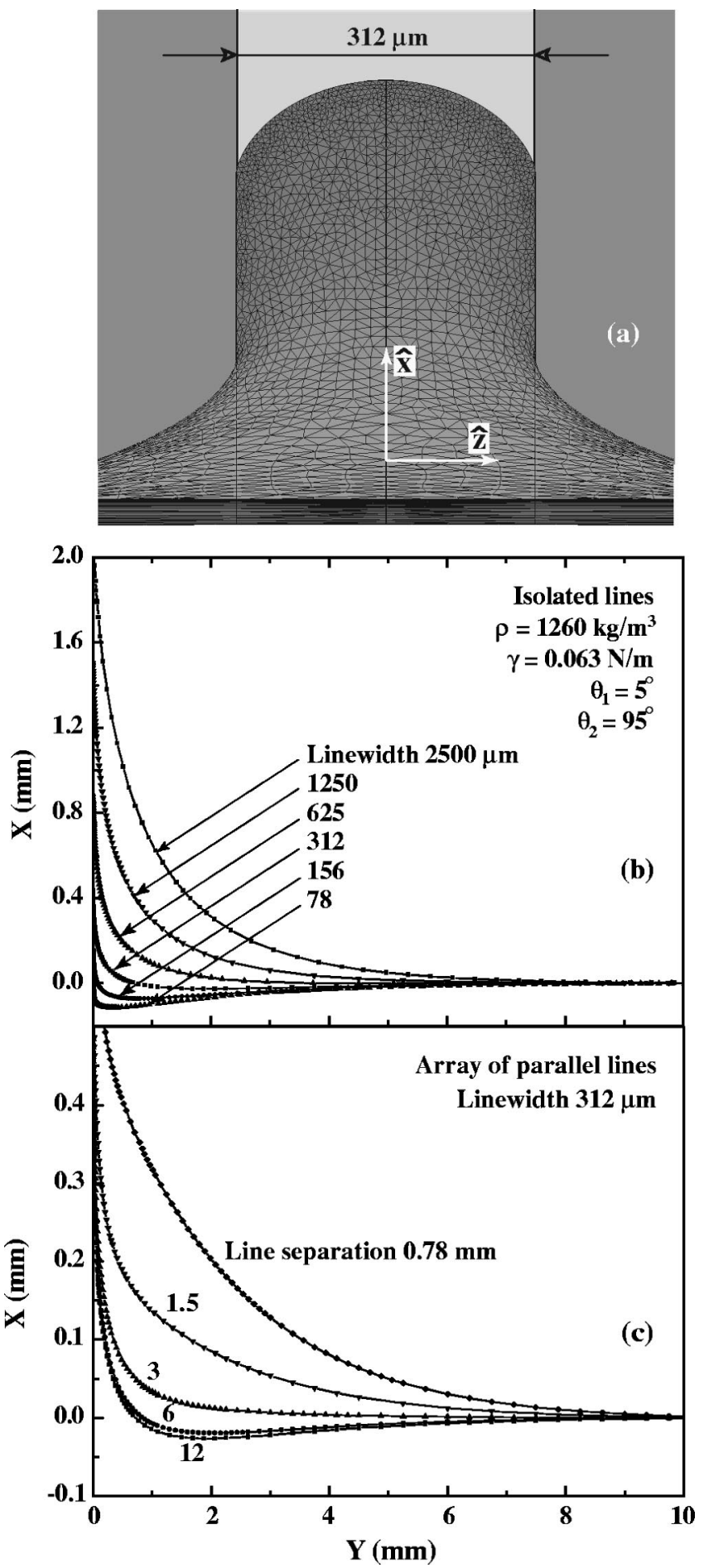

FIG. 2. (a) Liquid surface profile of a vertical $312 \mu \mathrm{m}$ wide hydrophilic line on a hydrophobic surface. (b) Linear meniscus profiles in the vertical symmetry plane of isolated lines for various linewidths ranging from 78.1 to $2500 \mu \mathrm{m}$. The linewidth increases by a factor of 2 from one curve to the next. (c) Linear meniscus profiles in the vertical symmetry plane of $312 \mu \mathrm{m}$ wide lines in a periodic array with line separations ranging from 0.78 to 12 $\mathrm{mm}$.

strip. Meniscus shapes for linewidths smaller than about 78 $\mu \mathrm{m}$ require excessive computational time since very fine surface triangulation is required to capture the detailed profile shape at the plane surface. However, the observed trend of a linear decrease in both the height of capillary rise and the 
vertical radius of curvature, remains valid down to much smaller linewidths provided the film coating is not so thin that disjoining forces become important.

Figure 2(a) shows an interesting detail involving the shape of the contact line as the liquid is forced to accommodate the presence two chemically different regions. The contact line precisely follows the vertical junction between the hydrophilic and hydrophobic portions over a fairly long distance. This point has been addressed by Boruvka and Neumann, ${ }^{25}$ who derived a semi-analytical solution of the Laplace-Young equation for the case of zero gravity. The contact angle is found to change from its hydrophobic to the hydrophilic value along this vertical segment. Schwartz and Garoff $^{26,27}$ performed similar studies of the shape of the liquid-solid contact lines on surfaces of mixed wettability.

For a dense array of vertical lines, the meniscus shape depends not only on the linewidth but also on the line separation. Plotted in Fig. 2(c) are the meniscus profiles along the vertical symmetry plane of a $312 \mu \mathrm{m}$ wide hydrophilic line for line separations ranging from $781 \mu \mathrm{m}$ to $12 \mathrm{~mm}$. The meniscus shape for 6 and $12 \mathrm{~mm}$ is practically identical. However, as the line separation decreases below the capillary length $(2.26 \mathrm{~mm})$, interference effects with neighboring lines increase the meniscus radius of curvature. This "cross-talk" leads to thicker film deposition on dense arrays than isolated lines. The increased liquid pickup can promote coalescence with neighboring lines to produce a film coating which covers not only the hydrophilic but the intervening hydrophobic portions as well.

The surface profile of an infinite line after dip coating corresponds to a section of a circular cylinder. The contact angle depends both on the natural contact angle on the hydrophilic strip and on the deposited liquid volume. If the natural contact angle is larger than the contact angle corresponding to the liquid pickup, the liquid will recede from part of the line and capillary breakup of the continuous line into two or more segments is very likely to occur. ${ }^{28}$ If the hydrophilic strips are not completely wetting and if the speed of withdrawal is smaller than the maximum contact line velocity ${ }^{29}$ very little or no liquid may be entrained on the hydrophilic parts. This is observed primarily for contaminated samples, where the contact angle is notably larger than $0^{\circ}$.

\section{Dip coating of a vertical hydrophilic strip: Scaling analysis for $\boldsymbol{h}_{\infty}$}

In what follows, we develop a model for the maximum film thickness of a hydrophilic liquid entrained on a hydrophilic strip surrounded by a hydrophobic coating. Consider the dip coating of a narrow, hydrophilic, vertical strip of half-width $W$ surrounded by a planar, hydrophobic surface as sketched in Fig. 2(a). A Cartesian coordinate system is defined with $\hat{x}$ the direction of plate withdrawal (i.e., the streamwise direction), $\hat{y}$ the direction normal to the plate, and $\hat{z}$ in the plane of the plate and normal to the direction of withdrawal. In deriving a relation for the maximum film thickness and liquid flux dragged upwards by the moving plate, we restrict attention to low capillary number flow $(\mathrm{Ca} \ll 1)$, small aspect ratios and negligible Bond number $\left(\mathrm{Bo}=\rho g h_{\infty}^{2} / \sigma \ll 1\right)$.

The flow profile exhibits three distinct regions. Far above the reservoir, the thickness of the entrained liquid film, $h_{\infty}$, is uniform and independent of $x$. In this region only viscous forces determine the upward flux of liquid since capillary pressure gradients vanish in the streamwise direction. At the reservoir the liquid surface assumes the shape of a static meniscus whose profile is strictly determined by the balance of capillary and hydrostatic pressures. The transition zone between these two regions, often called the dynamic meniscus regime, is governed by a balance between viscous and capillary forces. The film curvature in this region smoothly matches the value of the static meniscus curvature at the lower end and the film thickness approaches $h_{\infty}$ as $x$ $\rightarrow+\infty$.

Within the lubrication approximation, ${ }^{23}$ the NavierStokes equation governing the steady-state flow field in the transition zone reduces to

$$
-\frac{\partial p}{\partial x}+\mu \frac{\partial^{2} u}{\partial y^{2}}=0,
$$

which represents a balance between the capillary pressure gradient and the gradient in shear for nearly one-dimensional flow, based on the geometric requirement $\left(h_{\infty} / W\right)^{2} \ll 1$. The pressure, viscosity, and streamwise velocity are given by $p$, $\mu$, and $u$, respectively. This equation is solved subject to the no-slip condition at the solid-liquid interface

$$
u=U \quad \text { at } y=0,
$$

and vanishing shear stress at the air-liquid interface

$$
\mu \frac{\partial u}{\partial y}=0 \quad \text { at } y=h(x, z) .
$$

The pressure appearing in Eq. (3) is governed by the Laplace pressure

$$
\begin{aligned}
-\delta p & =\sigma \nabla_{s} \hat{n} \\
& =\sigma\left[\frac{h_{x x}\left(1+h_{z}^{2}\right)+h_{z z}\left(1+h_{x}^{2}\right)-2 h_{x} h_{z} h_{x z}}{\left(1+h_{x}^{2}+h_{z}^{2}\right)^{3 / 2}}\right],
\end{aligned}
$$

where $\nabla_{s}$ is the surface gradient operator and $\hat{n}$ the outward unit normal of the air-liquid interface. The subscripts refer to partial differentiation, i.e., $h_{x x}=\partial^{2} h / \partial x^{2}$.

Within the lubrication approximation, since $h_{x}^{2} \ll 1$ and $h_{z}^{2} \ll 1$, the earlier expression reduces to

$$
-\delta p \approx \sigma\left(h_{x x}+h_{z z}\right) .
$$

Substituting this expression for the pressure into Eq. (3), integrating twice with respect to $y$, and using the boundary conditions in Eqs. (4) and (5) yields the parabolic velocity profile

$$
u=U-\frac{\sigma}{\mu}\left(\frac{y^{2}}{2}-h y\right)\left(h_{x x x}+h_{x z z}\right) .
$$


The volumetric flow rate per unit width, which is controlled by the competition between the upward drag of fluid and the downward capillary drainage, is given by

$$
\begin{aligned}
Q & =\frac{1}{2 W} \int_{-W}^{+W} \int_{0}^{h} u d y d z \\
& =A h U+B \frac{\sigma h^{3}}{3 \mu}\left(h_{x x x}+h_{x z z}\right),
\end{aligned}
$$

where $A$ and $B$ are constants of order one resulting from the nonuniformity of the film profile in the $\hat{z}$ direction. Equation (10) assumes that the streamwise gradient of the capillary pressure is only weakly dependent on $z$. Far above the dynamic meniscus region, the entrained film thickness is uniformly flat in the direction of withdrawal. Under steady-state conditions, the flow rate emanating from the dynamic meniscus regime must therefore equal the flow rate as $x \rightarrow+\infty$, namely $Q=C h_{\infty} U$. The film thickness $h_{\infty}$ represents the steady-state height at the center of the strip (i.e., at $z=0$ ). The constant $C$ is a number of order one which results from averaging the film profile in the $z$ direction. (For example, $C=2 / 3$ for a liquid ribbon whose cross section is the arc of a circle.) Equating the two expressions for the flow rate yields a third order equation for the interface shape, $h(x, z)$

$$
\left(\frac{3 \mu U}{\sigma}\right) A h+B h^{3}\left(h_{x x x}+h_{x z z}\right)=\left(\frac{3 \mu U}{\sigma}\right) C h_{\infty} .
$$

Since the capillary pressure terms vanish and $h \rightarrow h_{\infty}$ as $x$ $\rightarrow \infty, A=C$ and Eq. (11) becomes

$$
h^{3}\left(h_{x x x}+h_{x z z}\right)=K(3 \mathrm{Ca})\left(h_{\infty}-h\right),
$$

where $K=A / B$ denotes a constant of order one.

This equation may be written in dimensionless form by introducing the set of reduced variables

$$
\eta=\frac{h}{h_{\infty}}, \quad \zeta=\frac{z}{W}, \quad \xi=\frac{x}{X_{s}},
$$

where $X_{s}$ is a characteristic length scale in the streamwise direction to be determined later from the matching of the curvature to the static meniscus.

Introducing these scaled variables into Eq. (11) gives

$$
\eta^{3}\left[\left(\frac{W}{X_{s}}\right)^{2} \eta_{\xi \xi \xi}+\eta_{\xi \zeta \zeta}\right]=K(3 \mathrm{Ca}) \frac{X_{s} W^{2}}{h_{\infty}^{3}}[1-\eta] .
$$

For the limiting case of a homogeneous flat plate, $K=1$ and $\left(W / X_{s}\right)^{2} \rightarrow \infty$, and Eq. (14) reduces to

$$
\eta^{3} \eta_{\xi \xi \xi}=\frac{X_{s}^{3}}{h_{\infty}^{3}}(3 \mathrm{Ca})[1-\eta],
$$

which determines the dependence of the streamwise length scale on the capillary number

$$
X_{s}=h_{\infty}(3 \mathrm{Ca})^{-1 / 3} .
$$

Using this relation in matching the film curvature at the lower end to the static meniscus yields the classical result given in Eq. (1).
In the general case where both the streamwise and transverse curvature contribute to the flow, there are two conditions which determine the scaling behavior of $X_{s}$, namely

$$
X_{s} \sim W \quad \text { and } X_{s} \sim \frac{h_{\infty}^{3}}{W^{2}}(\mathrm{Ca})^{-1} .
$$

The linear relation between $X_{s}$ and $W$ was also obtained from our simulations of the static meniscus discussed in Sec. III B. Equating these two expressions leads to the final result

$$
h_{\infty} \sim W(\mathrm{Ca})^{1 / 3} .
$$

In contrast to the Landau-Levich result in Eq. (1), the exponent associated with the capillary number is decreased from $2 / 3$ to $1 / 3$. In addition, the length scale controlling the deposited film thickness is not the capillary length but the channel half-width $W$. These two differences allow for deposition of much thinner coatings for comparable material constants and withdrawal speeds.

\section{EXPERIMENTAL RESULTS}

The key variables which control the film thickness deposited on a homogeneous surface by dip coating include the speed of withdrawal, liquid viscosity, surface tension, and density. For micropatterned surfaces, additional variables like the angular orientation of the hydrophilic shapes with respect to the withdrawal direction, and the width and geometry of the dipped patterns affect the shape and thickness of the coating film. We investigate the influence of these variables next.

\section{A. Velocity}

Using optical interferometry, we measured the maximum film thickness of glycerol entrained on an isolated 49 $\mu \mathrm{m}$ wide and $4 \mathrm{~mm}$ long hydrophilic strip on a $1 \times 1 \mathrm{~cm}^{2}$ hydrophobic sample. The cross section of the entrained liquid ribbon forms a sector of a circle. The sample was clamped at one of the upper corners and the line completely immersed in the liquid bath prior to withdrawal. The experimental data for the maximum film height $h_{\infty}$ of glycerol entrained on a hydrophilic line versus the speed of withdrawal $U$ is shown in Fig. 3. The solid line indicates power law behavior of the form $h_{\infty} \sim U^{0.33 \pm 0.005}$ in excellent agreement with the theoretical prediction of Eq. (18). Lines corresponding to exponents of 0.32 and 0.34 are drawn for comparison.

\section{B. Angular orientation}

We have measured the entrained coating film as a function of the azimuthal sample orientation, where the substrate is held vertical but rotated about an axis normal to the sample. The sample was withdrawn at a speed of $40 \mu \mathrm{m} / \mathrm{s}$. The experimental data for the entrained film thickness are plotted in Fig. 4. The solid line serves as a guide to the eye. As can be seen, $h_{\infty}$ increases monotonically from about 1.5 $\mu \mathrm{m}$ for vertically oriented lines to approximately twice this value for horizontal lines. The azimuthal dependence is rather weak for the range of angles $0 \leqslant \varphi \leqslant 45^{\circ}$. This plateau 


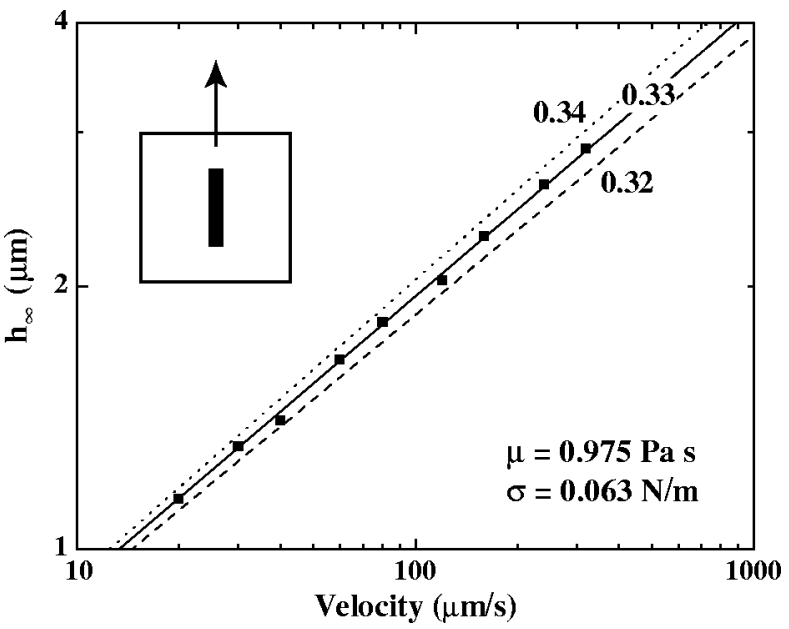

FIG. 3. Dependence of the height of entrained liquid lines on the speed of withdrawal $U$. The solid line represents a power law relation $h_{\infty} \sim U^{\beta}$ with $\beta=0.33$. The dashed and dotted lines represent equivalent power law relations with exponents 0.32 and 0.34 , respectively.

region is highly advantageous for printing purposes since the entrained film thickness is rather uniform across a broad range of angles.

We also investigated the influence of tilting the sample during withdrawal, i.e., the sample was rotated around an axis parallel to both the reservoir and the sample surface. Figure 1 suggests that the liquid volume pick-up increases on the upper side $(\alpha>0)$ and decreases on the lower side $(\alpha$ $<0)$ of the sample. Eberle and Reich have used this effect for the fabrication of optical filters. ${ }^{30}$ In our case, however, the vector of the sample velocity during withdrawal was not parallel to the sample surface as shown Fig. 1(a), but perpendicular to the surface of the liquid bath. Thus, the flow profile is different from the one which leads to the derivation of Eq. (2) and the results are not fully comparable. The experimental result for a tilt angle of $\alpha=30^{\circ}$ is an increase of $h_{\infty}$ by about $7 \%$ on the upper side for linewidths ranging from 46 to $65 \mu \mathrm{m}$ and a very small increase on the lower side. We attribute the increased pickup to the higher contact line ve-

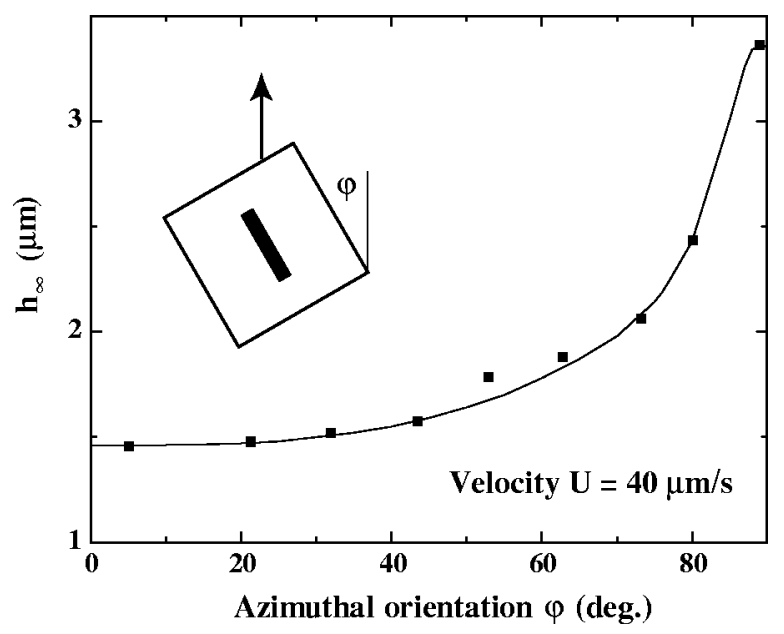

FIG. 4. Dependence of the height of the entrained liquid lines on the azimuthal sample orientation. The continuous line serves only as a guide to the eye.

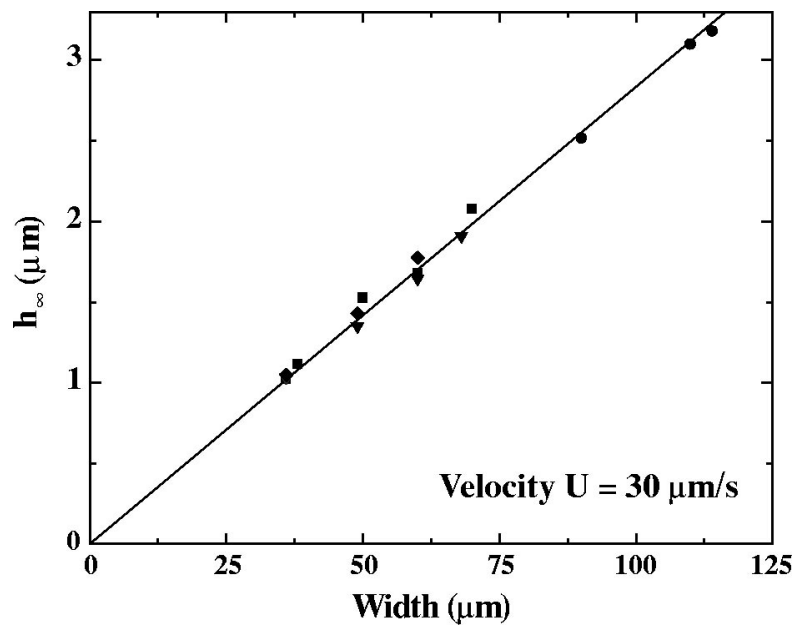

FIG. 5. Dependence of the height of the liquid lines on the width of the hydrophilic channels. Several different samples were investigated as indicated by the circles, squares, and triangles.

locity $U / \cos \alpha$ parallel to the sample surface, if the sample is tilted and withdrawn at the same vertical velocity $U$. The much smaller increase of $h_{\infty}$ on the lower side as compared to the upper side is qualitatively consistent with the reduced meniscus curvature on the upper side of the sample.

\section{Width and geometry}

To determine the dependence of the entrained volume on the linewidth of the hydrophilic strip, we investigated the dip-coating process for linewidths ranging from 40 to 110 $\mu \mathrm{m}$ withdrawn at a speed of $30 \mu \mathrm{m} / \mathrm{s}$. The experimental results for three different samples are shown in Fig. 5. Within experimental error, the maximum film thickness entrained on a vertical strip scales linearly with the linewidth.

Given the dependence of the maximum film thickness on the linewidth perpendicular to the direction of withdrawal, a sample withdrawn at an azimuthal angle $\varphi \neq 0$ will present an effective linewidth $W / \cos \varphi$. This larger width should entrain more liquid, which implies that hydrophilic lines oriented toward the horizontal will entrain a thicker coating than lines oriented closer to the vertical.

This is an undesirable consequence for printing applications where a uniform film height prior to printing is required. For patterns containing lines of arbitrary orientation, however, one could chemically micropattern a surface such as to segment lines into smaller rectangles to maintain a uniform film height throughout all hydrophilic regions. These hydrophilic patches would be separated by very narrow hydrophobic regions. Upon contact printing, these elements would coalesce and establish a continuous line as desired. We are exploring this tessellation procedure as a workaround to the orientation dependence of film height produced by dip coating as discussed above.

An additional problem involving nonuniform liquid pickup exists for the case of closed loops consisting of hydrophilic lines on a hydrophobic substrate. In Fig. 6(a) is shown a square loop consisting of four straight line segments $100 \mu \mathrm{m}$ in width and $1 \mathrm{~mm}$ in length. When such a loop is withdrawn at speeds ranging from 10 to $1000 \mu \mathrm{m} / \mathrm{s}$, the en- 


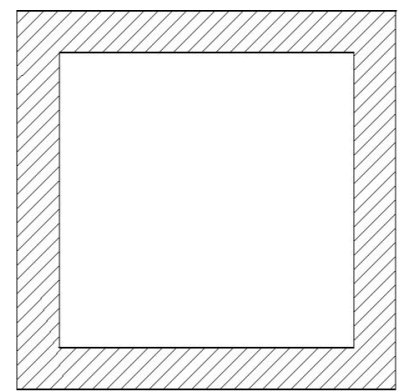

(a)

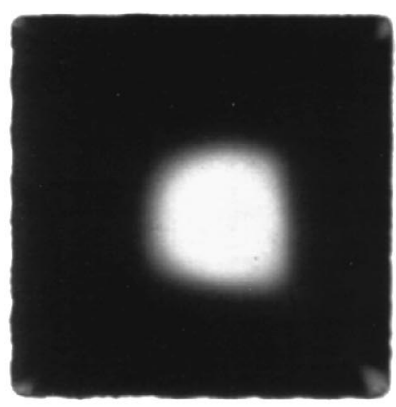

(b)

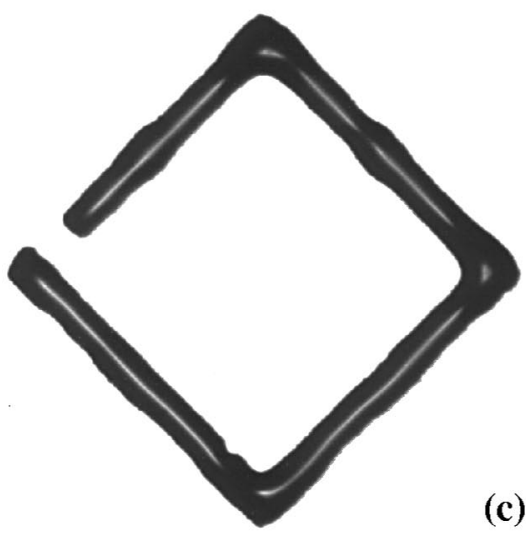

(c)

FIG. 6. (a) The designed pattern is a closed square loop, which (b) is filled completely with liquid during the dip-coating process. (c) This undesired effect can be avoided by breaking the loop in at least one position in the upper portion.

tire interior (hydrophobic) region becomes coated with a liquid film whose height exceeds the height entrained on an isolated hydrophilic strip of the same width and length.

As the square loop just exits the reservoir during the withdrawal process, a meniscus forms between the lower horizontal segment and the liquid bath. When this meniscus snaps off, the contact line recedes to the exterior edges of the loop thereby entrapping liquid throughout the hydrophobic interior as shown in Fig. 6(b). This behavior induces a complete loss of pattern fidelity which is detrimental for printing applications. Liquid entrapment of this sort occurs for loops of arbitrary azimuthal orientation withdrawn at speeds ranging over two orders of magnitude whose feature sizes span length scales ranging from microns to millimeters.

Figure 6(c) illustrates a solution to this problem. The loop shown consists of lines measuring $60 \mu \mathrm{m}$ in width and $600 \mu \mathrm{m}$ in length and is disconnected in the leftmost corner. This single interruption allows for a dewetting of the liquid, (a)
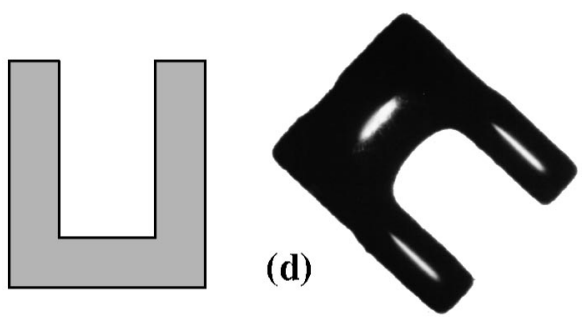

(b)

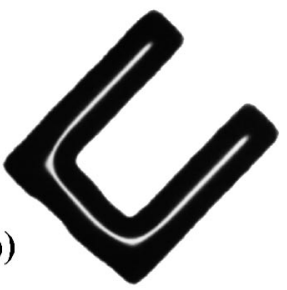

(c)

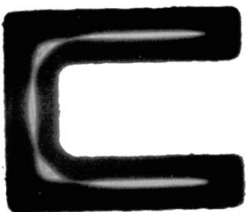

(e)

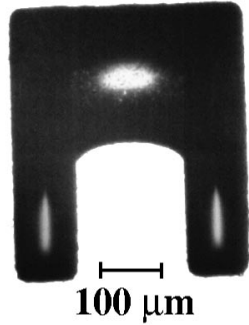

(f)

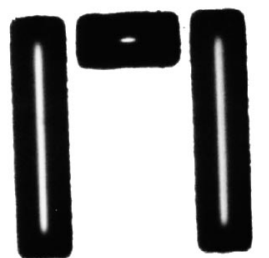

FIG. 7. The liquid volume entrained on a half-loop structure depends on its azimuthal orientation with respect to the direction of withdrawal. (a) Designed pattern in an upright position, (b)-(e) experimental hydrophilic half loops dipped in various orientations with the same speed of withdrawal, (f) disconnected half loop. Whereas the half loop structure picks up much more liquid in the orientations shown in (d) and (e), a disconnection of the upper part of the half loop avoids the undesired entrapment of liquid in the hydrophobic interior region.

which would otherwise be trapped in the hydrophobic interior of the hydrophilic loop, as the liquid meniscus recedes from the loop region. Therefore, the topological solution to interior entrapment of liquid is to disconnect closed line segments.

The position of the broken or disconnected point in an otherwise closed loop is critical for the deposition process since the volume entrained depends strongly on the pattern orientation. In Fig. 7(a) is shown a sketch of a U-shaped open loop. If during the vertical withdrawal process, the loop assumes orientations as shown in (a)-(c) (in which $0 \leqslant \varphi$ $\leqslant 90^{\circ}$ ), the level of liquid pickup is approximately invariant. However, once rotated beyond this angle such that the continuous part of the half loop appears at the top as in Figs. 7(d) and 7(e), liquid once again becomes entrapped in the interior hydrophobic region as discussed earlier for a closed loop. The excess volume does not distribute evenly on the hydrophobic interior but recedes to the inner corners of the structure in order to minimize the overall surface curvature and contact energy. These liquid bulges would again induce a loss of pattern fidelity upon printing.

The solution to this problem rests with inducing multiple disconnection points. When the half loop is separated into three straight line segments as shown in Fig. 7(f), no liquid entrapment occurred. Furthermore, the liquid pickup on each separated line segment is not vastly different. The spacing 
between the lines can be made even smaller than that shown in Fig. 7(f) so that the individual lines coalesce into the designed half loop pattern upon printing.

\section{SUMMARY}

We have investigated theoretically and experimentally the selective deposition of liquids on hydrophilic regions, chemically defined on a hydrophobic surface, by means of dip coating. This technique allows precise deposition of liquid coatings with thicknesses in the micron range. We have studied the entrained film height as a function of the speed of withdrawal. Sample orientation and pattern geometry also strongly influence the level of liquid pickup. For the case of vertically oriented hydrophilic lines, we find excellent agreement between the predictions of our theoretical model and the experimental results. This model extends the classical result of Landau and Levich to the case of chemically micropatterned substrates. High fidelity between the chemical surface pattern and the entrained liquid structures may require design changes including pattern segmentation or the introduction of disconnection points. This solution resolves the issue of height nonuniformities critical for applications involving high-resolution wet printing.

\section{ACKNOWLEDGMENTS}

This project is funded by the Electronic Technology Office of the Defense Advanced Research Projects Agency as part of the Molecular Level Printing Program. The authors also gratefully acknowledge the Austrian Fonds zur Förderung der wissenschaftlichen Forschung for a postdoctoral fellowship (AAD) and the Eastman Kodak Corporation for a graduate fellowship (SMM). Dr. N. Pittet and Dr. C. Monnereau assisted with the assembly of the dip-coating apparatus.
${ }^{1}$ L. E. Scriven, Mater. Res. Soc. Symp. Proc. 121, 717 (1988).

${ }^{2}$ K. J. Ruschak, Annu. Rev. Fluid Mech. 17, 65 (1985).

${ }^{3}$ Liquid Film Coating, edited by S. F. Kistler and P. M. Schweizer (Chapman \& Hall, London, 1997).

${ }^{4}$ A. Kumar and G. M. Whitesides, Appl. Phys. Lett. 63, 2002 (1993).

${ }^{5}$ L. Libioulle, A. Bietsch, H. Schmid, B. Michel, and E. Delamarche, Langmuir 15, 300 (1999).

${ }^{6}$ L. Landau and B. Levich, Acta Physicochim. URSS 17, 42 (1942).

${ }^{7}$ V. Levich, Physicochemical Hydrodynamics (Prentice-Hall, Englewood Cliffs, NJ, 1962).

${ }^{8}$ B. M. Deryagin and S. M. Levi, Film Coating Theory (Focal Press Ltd., New York, 1964), and references therein.

${ }^{9}$ C. Gutfinger and J. A. Tallmadge, AIChE J. 11, 403 (1965).

${ }^{10}$ D. A. White and J. A. Tallmadge, Chem. Eng. Sci. 20, 33 (1965).

${ }^{11}$ C. Y. Lee and J. A. Tallmadge, AIChE J. 18, 858 (1972).

${ }^{12}$ C. Y. Lee and J. A. Tallmadge, AIChE J. 19, 403 (1973).

${ }^{13}$ R. P. Spiers, C. V. Subbaraman, and W. L. Wilkinson, Chem. Eng. Sci. 29, 389 (1974).

${ }^{14}$ S. D. R. Wilson, J. Eng. Math. 16, 209 (1982).

${ }^{15}$ P. Tanguy, M. Fortin, and L. Choplin, Int. J. Numer. Methods Fluids 4, 441 (1984); 4, 459 (1984).

${ }^{16}$ O. Reglat, R. Labrie, and P. A. Tanguy, J. Comput. Phys. 109, 238 (1993).

${ }^{17}$ P. R. Schunk, A. J. Hurd, and C. J. Brinker, in Liquid Film Coating, edited by S. F. Kistler and P. M. Schweizer (Chapman \& Hall, London, 1997).

${ }^{18}$ D. Quéré, Annu. Rev. Fluid Mech. 10, 2790 (1994).

${ }^{19}$ H. A. Biebuyck and G. M. Whitesides, Langmuir 31, 347 (1999).

${ }^{20}$ D. Qin, Y. Xia, B. Xu, H. Yang, C. Zhu, G. M. Whitesides, Adv. Mater. 11, 1433 (1999).

${ }^{21}$ H. G. Braun and E. Meyer, Thin Solid Films 345, 222 (1999).

${ }^{22}$ Handbook of Chemistry and Physics, edited by R. C. Weast and M. J. Astle (Chemical Rubber Corp., Boca Raton, FL, 1982).

${ }^{23}$ R. F. Probstein, Physicochemical Hydrodynamics: An Introduction (Wiley, New York, 1994).

${ }^{24}$ We used the software package Surface Evolver for our simulations, which was developed by Kenneth Brakke of Susquehanna University, Selinsgrove, PA. For a review see K. Brakke, Exp. Math. 1, 141 (1992).

${ }^{25}$ A. L. Boruvka and W. Neumann, J. Colloid Interface Sci. 65, 315 (1978).

${ }^{26}$ L. W. Schwartz and S. Garoff, Langmuir 1, 219 (1985).

${ }^{27}$ L. W. Schwartz and S. Garoff, J. Colloid Interface Sci. 106, 422 (1985).

${ }^{28}$ A. A. Darhuber, S. M. Troian, S. M. Miller, and S. Wagner, J. Appl. Phys. 87, 7768 (2000).

${ }^{29}$ J. G. Petrov and R. V. Sedev, Colloids Surface 13, 313 (1985).

${ }^{30}$ A. Eberle and A. Reich, J. Non-Cryst. Solids 218, 156 (1997). 